\title{
Novelty-Induced Emotional Arousal Modulates Cannabinoid Effects on Recognition Memory and Adrenocortical Activity
}

\author{
Patrizia Campolongo*,1,7, Maria Morena ${ }^{1,7}$, Sergio Scaccianoce', Viviana Trezza ${ }^{2}$, Flavia Chiarotti ${ }^{3}$, \\ Gustav Schelling ${ }^{4}$, Vincenzo Cuomo' and Benno Roozendaal ${ }^{5,6}$ \\ 'Department of Physiology and Pharmacology, Sapienza University of Rome, P.le A. Moro 5, Rome, Italy; ${ }^{2}$ Department of Biology, University of \\ Roma Tre, Viale Marconi 446, Rome, Italy; ${ }^{3}$ Section of Neurotoxicology and Neuroendocrinology, Department of Cell Biology and Neuroscience, \\ Instituto Superiore di Sanità, Rome, Italy; ${ }^{4}$ Department of Anaesthesiology, Ludwig-Maximilians University, Munich, Germany; ${ }^{5}$ Department of \\ Cognitive Neuroscience, Radboud University Nijmegen Medical Centre, Nijmegen, The Netherlands; ${ }^{6}$ Donders Institute for Brain, Cognition and \\ Behaviour, Radboud University Nijmegen, Nijmegen, The Netherlands
}

Although it is well established that cannabinoid drugs can influence cognitive performance, the findings - describing both enhancing and impairing effects - have been ambiguous. Here, we investigated the effects of posttraining systemic administration of the synthetic cannabinoid agonist WIN55,212-2 (0.1, 0.3, or $1.0 \mathrm{mg} / \mathrm{kg})$ on short- and long-term retention of object recognition memory under two conditions that differed in their training-associated arousal level. In male Sprague-Dawley rats that were not previously habituated to the experimental context, WIN55,2 I2-2 administered immediately after a 3-min training trial, biphasically impaired retention performance at a I-h interval. In contrast, WIN55,2 I 2-2 enhanced I -h retention of rats that had received extensive prior habituation to the experimental context. Interestingly, immediate posttraining administration of WIN55,2I2-2 to non-habituated rats, in doses that impaired I-h retention, enhanced object recognition performance at a 24-h interval. Posttraining WIN55,2 I2-2 administration to habituated rats did not significantly affect $24-\mathrm{h}$ retention. In light of intimate interactions between cannabinoids and the hypothalamic-pituitary-adrenal axis, we further investigated whether cannabinoid administration might differently influence training-induced glucocorticoid activity in rats in these two habituation conditions. WIN55,212-2 administered after object recognition training elevated plasma corticosterone levels in non-habituated rats whereas it decreased corticosterone levels in habituated rats. Most importantly, following pretreatment with the corticosterone-synthesis inhibitor metyrapone, WIN55,2I 2-2 effects on I- and 24-h retention of non-habituated rats became similar to those seen in the low-aroused habituated animals, indicating that cannabinoid-induced regulation of adrenocortical activity contributes to the environmentally sensitive effects of systemically administered cannabinoids on short- and long-term retention of object recognition memory.

Neuropsychopharmacology (2013) 38, 1276-1286; doi:I0.1038/npp.2013.26; published online 20 February 2013

Keywords: cannabinoid receptors; emotional arousal; WIN55,2I2-2; corticosterone levels; hypothalamic-pituitary-adrenal (HPA) axis; glucocorticoids

\section{INTRODUCTION}

Extensive evidence indicates that cannabinoids, either administered exogenously or released from endogenous sites, have pronounced effects on learning and memory (Marsicano and Lafenetre, 2009; Wilson and Nicoll, 2002). The cannabinoid system consists of two types of G-proteincoupled receptors (CB1 and CB2 receptors), endogenous ligands, and enzymes involved in their synthesis and inactivation (Piomelli, 2003). Endogenous ligands for

*Correspondence: Dr P Campolongo, Department of Physiology and Pharmacology Sapienza, University of Rome, P.le A. Moro 5, Rome 00185, Italy, Tel: + 3906 499|2450, Fax: + 3906 49912480, E-mail: patrizia.campolongo@uniromal.it

${ }^{7}$ These authors contributed equally to this work. Received 22 October 20 I2; revised 5 January 20 I 3; accepted 9 January 2013; accepted article preview online 22 January 2013 cannabinoid receptors, that is, endocannabinoids, are synthesized on demand in an activity-dependent manner and released from postsynaptic neurons. They travel backward across the synapse to activate presynaptic CB1 receptors and modulate presynaptic functions (Piomelli, 2003). Cannabinoid signaling is crucial for certain forms of short- and long-term plasticity at both excitatory and inhibitory synapses (Deadwyler et al, 2007) and thereby contributes to various aspects of brain function, including learning and memory (Marsicano and Lafenetre, 2009). However, evidence indicates that the mnemonic consequence of cannabis exposure in humans does not always correspond to the effects observed in laboratory animals administered with cannabinoid compounds systemically or into discrete brain regions. For example, although it is well appreciated that cannabis use can impair short-term memory and executive function in humans (Pattij et al, 2008), not all 
preclinical studies, using WIN55,212-2 or other synthetic cannabinoid agonists, were able to confirm these findings (Baek et al, 2009; Clarke et al, 2008; Kosiorek et al, 2003; Schneider et al, 2008; Suenaga and Ichitani, 2008). Cannabinoid effects on long-term memory in humans did not receive much attention and findings of preclinical studies are not unequivocal, independent of the pharmacodynamic properties of the drug used. Whereas some animal studies indicate that systemic or intracranial administration of cannabinoid agonists impairs the encoding and consolidation of long-term memory processing (Barros et al, 2004; Robinson et al, 2008), enhancing effects are reported as well (De Oliveira Alvares et al, 2008). Although such discrepancies are not unusual in memory research, the factors contributing to these conflicting findings are yet poorly understood.

Emerging evidence indicates that cannabinoid drugs can induce distinct and even opposite effects on anxiety and several other behaviors, depending on the aversiveness of the environmental context (Campolongo et al, 2012; Carlin et al, 1972; Haller et al, 2009; Szuster et al, 1988; Zanettini et al, 2011). Based on these findings, we hypothesized that the level of emotional arousal that is associated with a training experience might also be a primary factor in determining the outcome of cannabinoid administration on learning and memory. Therefore, in the present study we investigated the effects of cannabinoid administration on both short- and long-term object recognition memory under two experimental conditions that differed with respect to their training-associated arousal level. By employing a previously described procedure (Okuda et al, 2004), one group of rats was not habituated to the training context, whereas the other group was extensively habituated to the experimental apparatus to decrease its noveltyinduced stress response during the training trial. The cannabinoid receptor agonist WIN55,212-2 was administered intraperitoneally to either extensively habituated or not previously habituated rats immediately after the object recognition training trial. In the first experiment, retention was tested $1 \mathrm{~h}$ after the training trial to assess possible cannabinoid effects on short-term cognitive performance. In the second experiment, we investigated cannabinoid effects on long-term object recognition memory by assessing retention $24 \mathrm{~h}$ after the training trial and drug treatment.

In addition to direct actions in the brain, cannabinoids are crucially involved in regulating hypothalamic-pituitaryadrenocortical (HPA) axis activity (Atsak et al, 2012b; Campolongo et al, 2009; Di et al, 2003; Hill et al, 2010b), shaping the corticosterone response to stressful stimulation. As it is well established that glucocorticoids hormones are potent modulators of learning and memory (Campolongo and Roozendaal, 2011; Okuda et al, 2004; Roozendaal, 2002; Roozendaal et al, 2006b; Schwabe et al, 2012), we next investigated whether the neuroendocrine consequence of cannabinoid administration on plasma corticosterone levels contributes to the environmentally sensitive effects of systemically administered WIN55,212-2 on object recognition memory. We first investigated whether WIN55,212-2 administration after object recognition training differentially affected the release of endogenous corticosterone in rats in the two habituation conditions. Further, to assess whether this cannabinoid-induced shaping of the corticosterone response plays a role in regulating the memorymodulatory influence of WIN55, 212-2, we examined whether pharmacological suppression of corticosterone synthesis with metyrapone altered the effects of WIN55,212-2 administration on both short- and long-term retention of object recognition memory.

\section{MATERIALS AND METHODS}

\section{Animals}

Male adult Sprague-Dawley rats $(350-450 \mathrm{~g}$ at the time of training, Charles River Laboratories, Italy) were kept individually in an air-conditioned colony room (temperature: $21 \pm 1{ }^{\circ} \mathrm{C}$; relative humidity: $60 \pm 10 \%$; lights on from 0700 to 1900 hours). Pellet food and water were available ad libitum. Training and testing were performed during the light phase of the cycle between 1000 and 1400 hours. All procedures involving animal care and treatments were in accordance with the guidelines released by the Italian Ministry of Health (D.L. 116/92) and the European Communities Council Directive of 24 November 1986 (86/ 609/EEC).

\section{Drug Treatment}

The cannabinoid receptor agonist WIN55,212-2 $(\mathrm{R}(+)$ [2,3-dihydro-5-methyl-3-[(morpholinyl) methyl] pyrolol [1,2,3-de]-1,4-benzoxazin-yl]-(1-naphthalenyl) methanone mesylate; $0.1, \quad 0.3$, and $1.0 \mathrm{mg} / \mathrm{kg}$; Sigma-Aldrich) was administered intraperitoneally in a volume of $1.0 \mathrm{ml} / \mathrm{kg}$ immediately after the training trial. Prior findings indicate that WIN55,212-2, after intraperitoneal administration, crosses the blood-brain barrier (Saghafi et al, 2011). For adrenocortical suppression, the $11 \beta$-hydroxylase inhibitor metyrapone (2-methyl-1,2-di-3-pyridyl-1-propanone; $35 \mathrm{mg} /$ $\mathrm{kg}$; Sigma-Aldrich) was injected in a volume of $2.0 \mathrm{ml} / \mathrm{kg}$ $40 \mathrm{~min}$ before the training trial. Metyrapone is a selective inhibitor of glucocorticoid synthesis in animals and humans (Schimmer and Parker, 2001). It blocks the conversion of the corticosterone precursor deoxycorticosterone in the adrenal cortex, thereby preventing the release of endogenous corticosterone into the bloodstream (Strashimirov and Bohus, 1966). All drugs were dissolved in 5\% polyethylene glycol, 5\% Tween- 80 , and $90 \%$ saline. The vehicle solution contained $5 \%$ polyethylene glycol and 5\% Tween-80 in saline only.

\section{Object Recognition Task}

The experimental apparatus was a gray open-field box (in $\mathrm{cm}, 40$ wide $\times 40$ deep $\times 40$ high) with the floor covered with sawdust, positioned in a dimly illuminated room. The objects to be discriminated were transparent glass vials $(5.5 \mathrm{~cm}$ diameter and $5 \mathrm{~cm}$ height) and white glass light bulbs $(6 \mathrm{~cm}$ diameter and $11 \mathrm{~cm}$ length).

All rats were handled twice per day for 1 min each for 7 days preceding the training day. The rats were divided into two groups. One group of rats was not habituated to the experimental apparatus (WITHOUT-habituation condition), whereas the other group was extensively habituated to the experimental context (WITH-habituation condition) 
to decrease their novelty stress to the apparatus during the training trial. During habituation, the rats were allowed to explore the apparatus in the absence of objects twice per day for 3 min each for 7 days (Okuda et al, 2004).

On the training trial, each rat was individually placed in the experimental apparatus at the opposite end from the objects. The rat was allowed to explore two identical objects (A1 and A2) for $3 \mathrm{~min}$, then was removed from the apparatus and, after drug treatment, returned to its home cage. To avoid the presence of olfactory trails, sawdust was stirred and the objects were cleaned with $70 \%$ ethanol after each trial. Rat's behavior was recorded by using a video camera positioned above the experimental apparatus. Exploration of an object was defined as pointing the nose to the object at a distance of $<1 \mathrm{~cm}$ and/or touching it with the nose. Turning around or sitting on an object was not considered as exploration. The time spent exploring the two objects was taken as a measure of object exploration, and exploratory behavior of the experimental apparatus was analyzed by the total number of rearings and crossings. For crossings, the floor of the apparatus was divided into four imaginary squares and the total number of crossings between squares was determined. Retention was tested either 1 or $24 \mathrm{~h}$ after the training trial. On the retention test trial, one copy of the familiar object (A3) and a new object (B) were placed in the same location as stimuli during the training trial. All combinations and locations of objects were used to reduce potential biases due to preference for particular locations or objects. Each rat was placed in the apparatus for $3 \mathrm{~min}$, and its behavior was recorded. Videos were analyzed by a trained observer who was unaware of treatment condition. The time spent exploring each object and the total time spent exploring both objects were recorded. To analyze cognitive performance, a discrimination index was calculated as the difference in time exploring the novel and the familiar object, expressed as the percentage ratio of the total time spent exploring both objects.

\section{Plasma Corticosterone Levels}

Corticosterone levels were determined in parallel groups of rats in the WITHOUT-habituation and WITH-habituation condition and in rats that were handled (twice per day for 7 days) but not trained. For the last experiment, the corticosterone-synthesis inhibitor metyrapone was injected $40 \mathrm{~min}$ prior to the training trial. As novelty stimulation triggers an HPA-axis response that leads to a corticosterone plasma peak at $15-30 \mathrm{~min}$ and returns to baseline by 60-90 min (Grota et al, 1997), rats were killed $30 \mathrm{~min}$ after training and WIN55,212-2 administration. Trunk blood was collected after decapitation and samples were centrifuged at $1900 \mathrm{~g}$ for $20 \mathrm{~min}$ at $4{ }^{\circ} \mathrm{C}$. Plasma was stored at $-80^{\circ} \mathrm{C}$ and analyzed for corticosterone using ELISA kits (Assay designs, Ann Arbor, MI, USA; IDS, Boldon, Tyne and Wear, UK) according to the manufacturer's instructions.

\section{Statistics}

All data are expressed as mean \pm SEM. Data were analyzed by one- or two-way ANOVA, followed by Tukey's post hoc comparison tests or paired or unpaired Student's $t$-tests, when appropriate. One-sample $t$-tests were used to determine whether the discrimination index was different from zero. A probability level of $<0.05$ was accepted as statistically significant. Fourteen rats were removed from statistical analyses because they showed a total exploration time of $<10 \mathrm{~s}$ on either training or testing. Prior findings indicate that such rats do not adequately acquire the task (Okuda et al, 2004).

\section{RESULTS}

Posttraining WIN55,212-2 Induces Opposite effects on 1-h Retention of Object Recognition Memory of Rats in the WITHOUT-Habituation and WITH-Habituation

\section{Condition}

This experiment investigated whether immediate posttraining injection of the cannabinoid receptor agonist WIN55,212-2 altered short-term performance on an object recognition task and whether this WIN55,212-2 effect was influenced by prior habituation to the experimental context.

Training trial. Two-way ANOVA for total exploration time of the two identical objects on the training trial revealed a significant habituation condition effect $\left(\mathrm{F}_{(1,88)}=11.46, \quad P=0.001\right)$, but no differences between posttraining drug groups or an interaction between habituation condition and later drug treatment. Rats in the WITHOUT-habituation condition showed significantly less total exploration of the two objects than rats in the WITH-habituation condition $\left(t_{94}=-3.37, \quad P=0.001\right.$; Figure 1a). In contrast, examination of rats' exploratory behavior of the training apparatus during the training trial indicated that the rats in the WITHOUT-habituation condition explored the experimental apparatus more than the rats in the WITH-habituation condition. Figure $1 \mathrm{~b}$ and $\mathrm{c}$ shows that the number of crossings and rearings were significantly higher in rats in the WITHOUT-habituation condition than that in rats in the WITH-habituation condition $\left(t_{94}=4.33, P<0.0001\right.$ for crossings, $t_{94}=3.36$, $P=0.001$ for rearings).
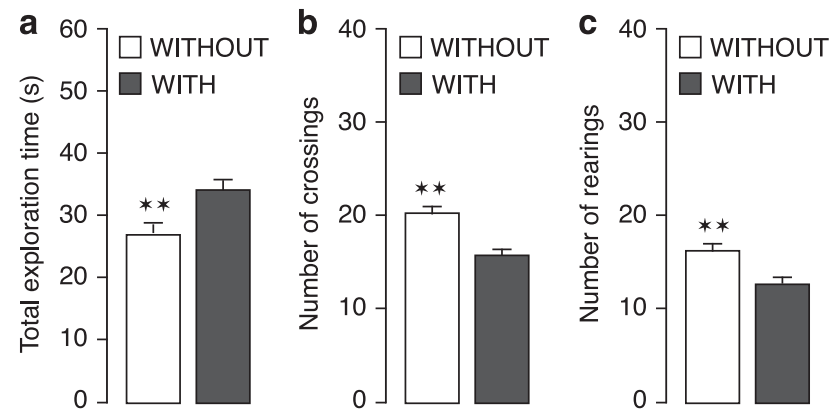

Figure I Behavioral effects during object recognition training in rats in the WITHOUT-habituation and WITH-habituation conditions. Rats in the WITHOUT-habituation (WITHOUT) condition spent significantly less time exploring the two identical objects during the training trial as compared with rats in the WITH-habituation (WITH) condition (a). Conversely, rats in the WITHOUT-habituation condition showed a higher number of crossings (b) and rearings (c) during training as compared with rats in the WITH-habituation condition. $* * P<0.0$ I vs the WITH-habituation group. Data are expressed as mean \pm SEM ( $n=48$ per group). 
Retention trial. One-sample $t$-tests revealed that the discrimination index of vehicle-treated rats was significantly different from zero in both the WITHOUT-habituation $\left(t_{11}=4.27, P=0.001\right)$ and WITH-habituation condition $\left(t_{11}=2.15, P=0.05\right)$, indicating that rats in both conditions discriminated the novel object at the 1-h retention interval. As shown in Figure 2, WIN55,212-2 administered immediately after the 3-min training trial, induced opposite effects on 1 -h retention performance of rats in the WITHOUThabituation and WITH-habituation conditions. Two-way ANOVA for discrimination index revealed no main effect of either drug treatment or habituation condition, but revealed a statistically significant interaction between these two factors $\left(\mathrm{F}_{(3,88)}=8.60, P<0.0001\right)$. Post hoc analysis indicated that the $0.3 \mathrm{mg} / \mathrm{kg}$ dose of WIN55,212-2, but not lower or higher doses, significantly decreased the discrimination index of rats in the WITHOUT-habituation condition $(P<0.05 ;$ Figure 2a). In contrast, the same dose of WIN55,212-2 increased the discrimination index of rats in the WITH-habituation condition $(P<0.05$; Figure $2 \mathrm{~b})$. Twoway ANOVA for total exploration time of the two objects during the retention trial revealed no statistically significant effects of habituation condition, drug treatment or of the interaction between these two factors. Rats' exploratory behavior of the apparatus during the test trial also did not differ between rats in the WITHOUT-habituation and WITH-habituation condition (Table 1).

Posttraining WIN55,212-2 Enhances 24-h Retention of Object Recognition Memory of Rats in the WITHOUTHabituation but not in the WITH-Habituation Condition

This experiment examined, in separate groups of rats, whether immediate posttraining injection of WIN55,212-2 influenced long-term performance on an object recognition task and whether this WIN55,212-2 effect was also influenced by prior habituation to the experimental context.

Training trial. The pattern of effects on the training trial was highly comparable to that observed in the first experiment. Two-way ANOVA for total exploration time


Figure 2 Effect of posttraining administration of WIN55,2 I2-2 on I-h retention of rats in the WITHOUT- and WITH-habituation conditions. Posttraining administration of WIN55,2 $12-2$ (WIN, $0.3 \mathrm{mg} / \mathrm{kg}$, i.p.) impaired I-h retention of object recognition memory of rats in the WITHOUThabituation condition (a) but enhanced I-h retention of rats in the WITHhabituation condition (b). ${ }^{*} P<0.05$ vs the corresponding vehicle control group. Data are expressed as mean \pm SEM ( $n=12$ per group). of the two objects on the training trial revealed a significant habituation condition effect $\left(\mathrm{F}_{(1,88)}=4.04, P=0.05\right)$, but no differences between posttraining drug groups or an interaction between habituation condition and posttraining drug treatment. Rats in the WITHOUT-habituation condition explored the two objects significantly less $(23.7 \pm 1.3 \mathrm{~s})$ than did rats in the WITH-habituation condition $(28.2 \pm 1.9 \mathrm{~s})$ $\left(t_{94}=-2.05, P<0.05\right)$. Also, as found in the first experiment, rats in the WITHOUT-habituation condition showed significantly more exploration of the experimental apparatus than did rats in the WITH-habituation condition, as indicated by a higher number of crossings $\left(t_{94}=2.89\right.$, $P=0.005)$ and rearings $\left(t_{94}=2.92, P=0.004\right)$.

Retention trial. As expected, after a 3-min training trial, rats of both vehicle groups did not express long-term retention of the familiar object: One-sample $t$-tests revealed no preference for the novel object in vehicle-treated rats in either the WITHOUT-habituation or WITH-habituation condition. As shown in Figure 3, posttraining WIN55,2122 induced different effects on object recognition memory of rats in the WITHOUT-habituation and WITH-habituation groups at the 24-h retention interval. Two-way ANOVA for discrimination index indicated no main effect of either drug treatment or habituation condition, but revealed a statistically significant drug treatment $\times$ habituation condition interaction $\left(\mathrm{F}_{(3,88)}=5.65, \quad P=0.001\right)$. WIN55,212-2 improved $24-\mathrm{h}$ retention of rats in the WITHOUT-habituation condition in an inverted $U$-shape relationship $\left(\mathrm{F}_{(3,49)}=3.81\right.$, $P=0.02$; Figure $3 \mathrm{a}$ ), without significantly affecting $24-\mathrm{h}$ retention of rats in the WITH-habituation condition $\left(\mathrm{F}_{(3,39)}=2.49, \quad P=0.07\right.$ Figure $\left.3 \mathrm{~b}\right)$. Post hoc analysis indicated that the $0.3 \mathrm{mg} / \mathrm{kg}$ dose of WIN55,212-2, but not lower or higher doses, administered to rats in the WITHOUT-habituation condition increased the discrimination index as compared with vehicle controls $(P<0.05)$. Twoway ANOVA for total exploration time of the two objects during the retention trial revealed a significant habituation

Table I Exploratory Behavior of Rats in the WITHOUT- and WITH-Habituation Conditions at the I-h Retention Test

\begin{tabular}{|c|c|c|c|}
\hline & $\begin{array}{c}\text { Total object } \\
\text { exploration time (s) }\end{array}$ & $\begin{array}{l}\text { Number of } \\
\text { crossings }\end{array}$ & $\begin{array}{c}\text { Number of } \\
\text { rearings }\end{array}$ \\
\hline \multicolumn{4}{|l|}{ WITHOUT } \\
\hline Vehicle & $28.2 \pm 3.3$ & $14.0 \pm 1.3$ & $17.5 \pm 2.2$ \\
\hline WIN 0.1 & $21.3 \pm 2.0$ & $13.5 \pm 1.2$ & $15.5 \pm 2.0$ \\
\hline WIN 0.3 & $23.2 \pm 1.8$ & $11.0 \pm 1.5$ & $15.9 \pm 3.2$ \\
\hline WIN I.0 & $22.8 \pm 3.7$ & $13.4 \pm 2.4$ & $14.9 \pm 3.0$ \\
\hline \multicolumn{4}{|l|}{ WITH } \\
\hline Vehicle & $25.9 \pm 3.3$ & $15.0 \pm 1.9$ & $15.9 \pm 1.5$ \\
\hline WIN 0.1 & $25.2 \pm 3.3$ & $14.6 \pm 1.5$ & $19.9 \pm 3.2$ \\
\hline WIN 0.3 & $30.8 \pm 4.0$ & $\mid 4.3 \pm 1.7$ & $15.3 \pm 1.7$ \\
\hline WIN 1.0 & $30.9 \pm 3.9$ & $12.6 \pm 1.5$ & $\mid 5.1 \pm 2.2$ \\
\hline
\end{tabular}

Total time spent exploring the two objects (in seconds) and the number of crossings and rearings of all groups in the WITHOUT- and WITH-habituation conditions. Results are expressed as mean \pm SEM ( $n=12$ per group). 
condition effect $\left(\mathrm{F}_{(1,88)}=6.67, P=0.01\right)$, but no drug treatment effect or interaction between both factors (Table 2). Comparable to the findings of the first experiment, rats' exploratory behavior of the experimental apparatus during the retention test trial did not differ between rats in the WITHOUT-habituation and the WITHhabituation conditions (Table 2).

Posttraining WIN55,212-2 Induces Opposite Effects on Plasma Corticosterone Levels in Rats in the WITHOUTHabituation and WITH-Habituation Condition

Figure 4 shows plasma corticosterone levels of parallel groups of trained rats in the WITHOUT-habituation or WITH-habituation condition, as assessed $30 \mathrm{~min}$ after the training trial and WIN55,212-2 injection. Another group of
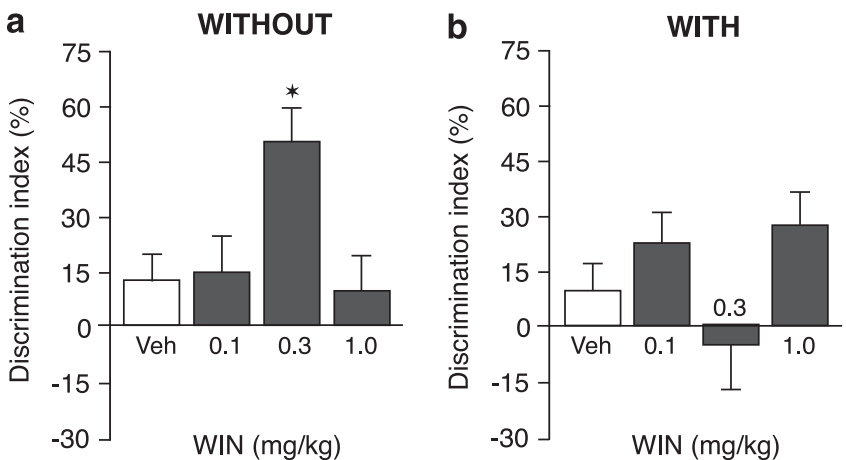

Figure 3 Effect of posttraining administration of WIN55,2 I 2-2 on 24-h retention of rats in the WITHOUT- and WITH-habituation conditions. Posttraining administration of WIN55,2/2-2 (WIN, $0.3 \mathrm{mg} / \mathrm{kg}$, i.p.) enhanced 24-h retention of object recognition memory of rats in the WITHOUT-habituation condition (a) and did not significantly affect 24-h retention of rats in the WITH-habituation condition (b). $* P<0.05$ vs the corresponding vehicle control group. Data are expressed as mean \pm SEM ( $n=10-14$ per group).

Table 2 Exploratory Behavior of Rats in the WITHOUT- and WITH-Habituation Conditions at the 24-h Retention Test

\begin{tabular}{cccc}
\hline & $\begin{array}{c}\text { Total object } \\
\text { exploration time (s) }\end{array}$ & $\begin{array}{c}\text { Number of } \\
\text { crossings }\end{array}$ & $\begin{array}{c}\text { Number of } \\
\text { rearings }\end{array}$ \\
\hline WITHOUT & $18.0 \pm 1.7$ & $13.2 \pm 1.4$ & $12.3 \pm 1.2$ \\
Vehicle & $19.9 \pm 2.1$ & $13.4 \pm 1.2$ & $12.2 \pm 0.9$ \\
WIN 0.1 & $15.5 \pm 0.7$ & $13.8 \pm 1.6$ & $14.3 \pm 1.1$ \\
WIN 0.3 & $22.6 \pm 2.3$ & $10.5 \pm 0.9$ & $11.8 \pm 1.2$ \\
WIN 1.0 & & & \\
& & & $12.9 \pm 1.2$ \\
WITH & $18.8 \pm 1.7$ & $11.0 \pm 1.1$ & $11.1 \pm 1.5$ \\
Vehicle & $26.0 \pm 3.9$ & $12.9 \pm 2.6$ & $13.3 \pm 2.6$ \\
WIN 0.1 & $24.1 \pm 2.3$ & $10.3 \pm 1.1$ & $12.3 \pm 2.2$ \\
WIN 0.3 & &
\end{tabular}

Total time spent exploring the two objects (in seconds) and the number of crossings and rearings of all groups in the WITHOUT- and WITH-habituation conditions. Results are expressed as mean \pm SEM ( $n=10-14$ per group). rats was administered WIN55,212-2, but was only handled and not trained (home cage). Concerning plasma corticosterone levels in rats treated with vehicle only, one-way ANOVA revealed a significant habituation condition effect $\left(\mathrm{F}_{(2,14)}=30.75, \quad P<0.0001\right)$. Post hoc comparison tests showed that plasma corticosterone levels in rats in the WITHOUT-habituation condition treated with vehicle were significantly higher than those in vehicle-injected rats in the WITH-habituation condition $(P<0.05)$, supporting the view that the habituation procedure efficiently suppressed the level of emotional arousal during the training trial. Further, plasma corticosterone levels in vehicle-treated trained rats in both the WITHOUT- and WITH-habituation conditions were significantly higher than those in non-trained vehicletreated rats $(P<0.01$ for both comparisons).

WIN55,212-2 induced opposite effects on plasma corticosterone levels in rats after object recognition training, depending on the level of emotional arousal at the time of drug administration. Two-way ANOVA revealed a significant habituation condition effect $\left(\mathrm{F}_{(2,56)}=41.19\right.$, $P<0.0001)$, drug treatment effect $\left(\mathrm{F}_{(3,56)}=9.58, P<0.0001\right)$ and interaction between both factors $\left(\mathrm{F}_{(6,56)}=4.78\right.$, $P=0.0005)$. Post hoc analysis indicated that the $1.0 \mathrm{mg} / \mathrm{kg}$ dose of WIN55,212-2 elevated plasma corticosterone levels in rats in the WITHOUT-habituation condition $(P<0.01)$. In contrast, the $0.3 \mathrm{mg} / \mathrm{kg}$ dose of WIN55,212-2, but not any of the other doses, decreased plasma corticosterone levels when administered to rats in the WITH-habituation condition $(P<0.05)$. WIN55,212-2 administration did not significantly alter plasma corticosterone levels in non-trained control rats.

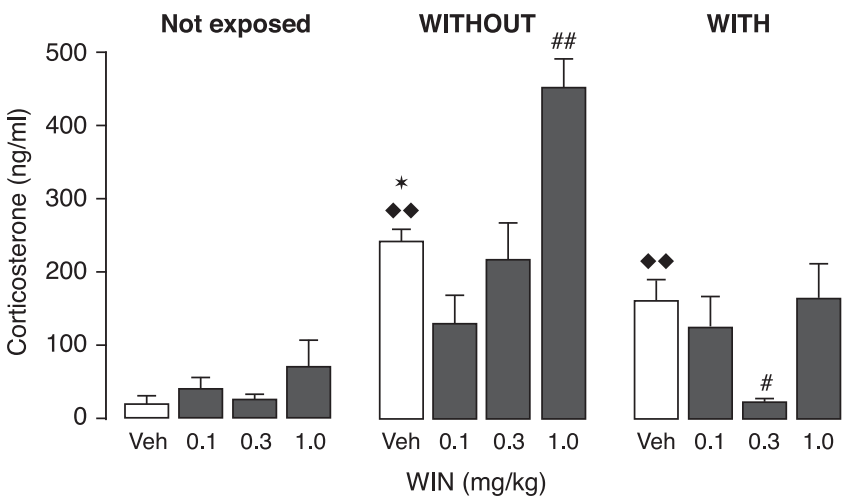

Figure 4 Effect of object recognition training and posttraining administration of WIN55,212-2 on plasma corticosterone levels in rats in the WITHOUT- and WITH-habituation conditions. Plasma corticosterone levels in rats in the WITHOUT-habituation condition treated with vehicle posttraining were significantly higher than those in vehicle-injected rats in the WITH-habituation condition, as assessed 30 min after training; $* P<0.05$ vs vehicle-treated rats in the WITH-habituation condition. Further, plasma corticosterone levels in vehicle-treated trained rats in both the WITHOUT- and WITH-habituation conditions were significantly higher than those in non-trained vehicle-treated rats; $\bullet P<0.01$ vs vehicle-treated not exposed rats. WIN55-2 I 2,2 (WIN, I mg/kg, i.p.) administered immediately after object recognition training increased plasma corticosterone levels in rats in the WITHOUT-habituation condition; ${ }^{\# \#} P<0.01$ vs the corresponding vehicle-treated control group. In contrast, WIN55,2I2-2 $(0.3 \mathrm{mg} / \mathrm{kg}$, i.p.) decreased plasma corticosterone levels in habituated rats; ${ }^{\#} P<0.05$ vs the corresponding vehicle-treated control group. Data are expressed as mean \pm SEM ( $n=4-7$ per group). 
Adrenocortical Suppression in Rats in the WITHOUTHabituation Condition Modifies the Effect of Posttraining WIN55,212-2 on Short- and Long-Term Object Recognition Memory

The findings described above indicate that WIN55,212-2 administered to rats in the two habituation conditions not only induces opposite effects on short- and long-term retention of object recognition memory but also on plasma corticosterone levels. To determine whether the WIN55,2122 effect on the corticosterone response contributes to how WIN55,212-2 influences object recognition memory, in the last experiment we investigated whether pharmacological suppression of adrenocortical activity with metyrapone (35 mg/kg, i.p.), administered to non-habituated rats $40 \mathrm{~min}$ prior to the training trial, altered the effects of posttraining WIN55,212-2 administration on short- and long-term object recognition memory.

Training trial. Two-way ANOVA for total exploration time of the two identical objects on the training trial revealed no significant metyrapone effect, no difference between posttraining WIN55,212-2 treatment groups or interaction between these two parameters. Two-way ANOVAs for the number of crossings and rearings on the training trial also did not reveal any significant metyrapone or later WIN55,212-2 treatment effect.

One-hour retention. As shown in Figure 5a, pretreatment of rats in the WITHOUT-habituation condition with metyrapone transformed the effect of posttraining WIN55,212-2 administration on 1-h retention performance into that of rats in the WITH-habituation condition. Twoway ANOVA for discrimination index indicated no main effect of metyrapone or WIN55,212-2 treatment, but revealed a significant metyrapone $\times$ WIN55,212-2 interaction effect $\left(\mathrm{F}_{(1,35)}=11.83, P=0.02\right)$. Comparable to the
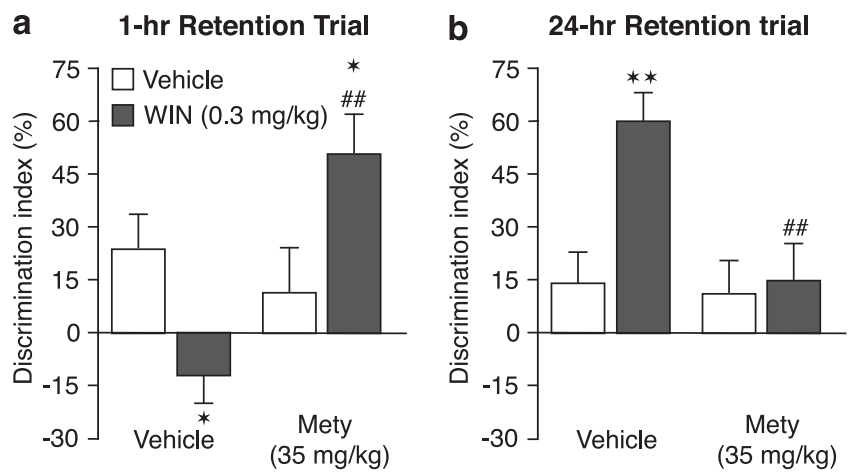

Figure 5 Effect of pretreatment with metyrapone on the effect of posttraining administration of WIN55,212-2 on I- and 24-h retention of rats in the WITHOUT-habituation condition. Metyrapone (Mety, $35 \mathrm{mg} / \mathrm{kg}$, i.p.) administered to rats in the WITHOUT-habituation condition 40 min before object recognition training reverted the impairing effect of posttraining WIN55,2I 2-2 (WIN, $0.3 \mathrm{mg} / \mathrm{kg}$, i.p.) on I-h retention (a) and the enhancing effect of WIN55,2I $2-2(0.3 \mathrm{mg} / \mathrm{kg}$, i.p.) on 24-h retention (b). Both I- and 24-h retention performance became very similar to those observed in low-aroused rats in the WITH-habituation condition (see Figures $2 b$ and $3 b$ ). $* P<0.05$; $* * P<0.01$ vs the corresponding vehicle group, ${ }^{\#} P<0.01$ vs the WIN55,2 1 2-2 alone group. Data are expressed as mean \pm SEM ( $n=9-12$ per group). findings shown in Figure 2a, WIN55,212-2 $(0.3 \mathrm{mg} / \mathrm{kg})$ administered alone to non-habituated rats immediately after the training trial significantly decreased the discrimination index relative to that of vehicle-treated rats $(P<0.05)$. However, in non-habituated rats pretreated with metyrapone, the same dose of WIN55,212-2 significantly increased the discrimination index $(P<0.05 v s$ vehicle) and thus highly resembled the WIN55,212-2 effect on 1-h retention as described above for rats in the WITHhabituation condition (see, Figure 2b). Metyrapone pretreatment by itself did not significantly alter the discrimination index. Total exploration time of the two objects and rats' exploratory behavior of the apparatus on the 1-h retention test did not differ between drug treatment groups (Table 3).

Twenty-four-hour retention. As shown in Figure 5b, pretreatment of non-habituated rats with metyrapone also transformed the effect of posttraining WIN55,212-2 on 24-h retention performance into that of rats in the WITHhabituation condition. Two-way ANOVA for discrimination index indicated no main effect of either metyrapone or WIN55,212-2 treatment, but revealed a significant metyrapone $\times$ WIN55,212-2 interaction effect $\left(\mathrm{F}_{(1,40)}=4.94\right.$, $P=0.03)$. Comparable to the findings shown in Figure $3 \mathrm{a}$, WIN55,212-2 $(0.3 \mathrm{mg} / \mathrm{kg})$ administration alone significantly increased the discrimination index of rats in the WITHOUT-habituation condition $(P<0.01)$. However, in rats pretreated with metyrapone, posttraining WIN55,212-2 administration did not significantly alter retention performance on the 24-h test trial, thus resembling the findings described above for rats in the WITH-habituation condition (see, Figure $3 \mathrm{~b}$ ). Metyrapone pretreatment alone did not significantly alter the discrimination index. Total exploration time of the two objects during the 24-h retention trial and rats' exploratory behavior of the apparatus did not differ between drug treatment groups (Table 3).

Table 3 Exploratory Behavior of Rats in the WITHOUTHabituation Condition Injected with Metyrapone and WIN55,2122 at the I- and 24-h Retention Tests

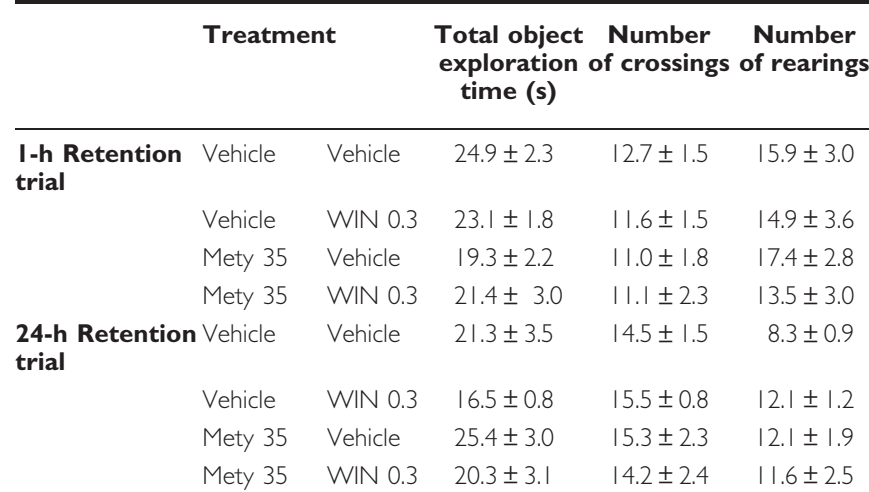

\footnotetext{
Total time spent exploring the two objects (in seconds) and the number of crossings and rearings of rats in the WITHOUT-habituation condition administered metyrapone (Mety, mg/kg, i.p.) 40 min prior to training and WIN55,2I 2-2 (WIN, mg/kg, i.p.) immediately posttraining. Results are expressed as mean $\pm \operatorname{SEM}(n=10-12$ per group).
} 
Plasma corticosterone levels. Table 4 shows the effect of metyrapone pretreatment and posttraining WIN55,212-2 administration on plasma corticosterone levels in rats in the WITHOUT-habituation condition, as assessed $30 \mathrm{~min}$ after object recognition training and WIN55,212-2 administration. Rats treated with metyrapone $40 \mathrm{~min}$ prior to the training trial had significantly lower plasma corticosterone levels than rats administered vehicle before training $\left(t_{8}=3.44, P=0.009\right)$. In rats pretreated with metyrapone, posttraining WIN55,212-2 administration did not significantly elevate plasma corticosterone levels.

\section{DISCUSSION}

Using a previously described habituation procedure, we show that the synthetic cannabinoid agonist WIN55,212-2 interacts with training-associated emotional arousal in influencing both short- and long-term object recognition memory. Furthermore, our findings indicate that the cannabinoid system shapes the response of the HPA-axis to emotional arousal and that this neuroendocrine action is importantly involved in determining how WIN55,212-2 might affect object recognition memory. WIN55,212-2, administered systemically immediately after object recognition training, biphasically impaired retention at a $1-\mathrm{h}$ interval in rats that were not habituated to the experimental context. In contrast, the same dose of WIN55,212-2 enhanced 1-h retention of rats that had reduced noveltyinduced emotional arousal because of extensive prior habituation to the experimental context. Additionally, immediate posttraining administration of WIN55,212-2 to non-habituated rats, in a dose that impaired 1-h retention, enhanced object recognition performance at a 24 -h interval. Posttraining WIN55,212-2 administration did not significantly affect 24 -h retention of habituated rats. This habituation procedure also produced opposite cannabinoid effects on training-induced HPA-axis activity. WIN55,212-2 administration after object recognition training elevated plasma corticosterone levels in rats that were not previously habituated to the experimental context, but decreased levels in habituated rats. Most importantly, as nonhabituated rats administered the corticosterone-synthesis inhibitor metyrapone prior to training and WIN55,212-2

Table 4 Plasma Corticosterone Levels in Rats in the WITHOUTHabituation Condition Injected with Metyrapone and WIN55,2 I2-2

\begin{tabular}{lll}
\hline Mety & WIN & Cort, $\mathbf{n g} / \mathbf{m l}$ \\
\hline Vehicle & Vehicle & $245.3 \pm 12.7$ \\
Mety 35 & Vehicle & $158.1 \pm 22.0 * *$ \\
Mety 35 & WIN 0.I & $190.0 \pm 47.8$ \\
Mety 35 & WIN 0.3 & $222.8 \pm 40.4$ \\
Mety 35 & WIN I.0 & $180.1 \pm 20.3$ \\
\hline
\end{tabular}

Plasma corticosterone (Cort) levels (mean \pm SEM) in $\mathrm{ng} / \mathrm{ml}$ in rats in the WITHOUT-habituation condition as assessed 30 min after the training trial. Metyrapone (Mety, $\mathrm{mg} / \mathrm{kg}$, i.p.), injected $40 \mathrm{~min}$ prior to training, reduced training-induced plasma corticosterone levels and prevented the increase in corticosterone plasma levels induced by posttraining WIN55,2 I2-2 (WIN, mg/kg, i.p.) administration. ${ }^{*} * P<0.01$ vs the vehicle control group ( $n=4-5$ per group). injection performed equally to extensively habituated rats on short- and long-term retention tests, these findings indicate that cannabinoid-induced regulation of adrenocortical activity contributes to the environmentally sensitive effects of systemically administered cannabinoids on shortand long-term retention of object recognition memory. Table 5 summarizes our findings of WIN55,212-2 on both short- and long-term recognition memory and on adrenocortical activity.

Clinical evidence indicates that cannabis use, either chronic consumption or acute intoxication, can impair both short-term memory and executive function (Pattij et al, 2008). Although some preclinical studies were able to confirm these findings (Baek et al, 2009; Kosiorek et al, 2003), the literature appears highly ambiguous (Clarke et al, 2008; Suenaga and Ichitani, 2008). In a previous study, we demonstrated that pretraining enhancement of endocannabinoid tone biphasically modified short-term retention of a recognition task in a spatial open field, and that this effect was likely influenced by the emotional state of the rat during the training trial (Campolongo et al, 2012). Here, we employed posttraining drug administration in order to more properly investigate the cognitive processes involved and found that the level of emotional arousal at encoding influenced the outcome of later administration of the cannabinoid agonist WIN55,212-2 on both short- and long-term retention of object recognition memory. As vehicle-control rats in both habituation conditions showed a similar preference for the novel object at the 1-h retention interval, it is unlikely that any difference in acquisition (ie, total exploration of the objects during the training trial) between the two groups underlies the differential effects. It seems most likely that a difference in the arousal level induced by the habituation procedure was critical. The finding that non-habituated rats displayed significantly higher levels of locomotion and rearing behavior during the training trial than habituated rats is consistent with previous evidence that exposure of rats to novel contexts induces changes in behavioral responses, including hyperlocomotion and increased rearing behavior (Okuda et al, 2004; van den Buuse et al, 2001). Novelty-induced arousal also activates stress hormone systems, including glucocorticoids (Emmert and Herman, 1999; Handa et al, 1994) and epinephrine (Feenstra et al, 2000; McQuade et al, 1999). Corroborating previous findings by Okuda et al (2004), we found that non-habituated rats had a significantly more

Table 5 Schematic Representation of the Effects of Posttraining WIN55,2 I2-2 on Recognition Memory

\begin{tabular}{lccc}
\hline $\begin{array}{l}\text { Habituation } \\
\text { condition }\end{array}$ & Treatment & $\begin{array}{c}\text { Short-term } \\
\text { memory effect }\end{array}$ & $\begin{array}{c}\text { Long-term } \\
\text { memory effect }\end{array}$ \\
\hline WITHOUT & WIN & $\downarrow$ & $\uparrow$ \\
WITH & WIN & $\uparrow$ & - \\
WITHOUT & WIN + Mety & $\uparrow$ & -
\end{tabular}

Schematic representation of the effects of posttraining WIN55,2 I2-2 (WIN, $0.3 \mathrm{mg} / \mathrm{kg}$, i.p.) either alone or together with the corticosterone-synthesis inhibitor metyrapone (Mety, $35 \mathrm{mg} / \mathrm{kg}$, i.p.) on I- and 24-h retention performance in rats in the WITHOUT-habituation and WITH-habituation conditions. 
pronounced training-induced increase in plasma corticosterone levels than habituated rats. Although these behavioral and endocrine measures clearly indicate that our habituation procedure efficiently reduced the level of emotional arousal during the training session, we cannot exclude the possibility that it is the stress history and not the level of emotional arousal per se that may be influencing cannabinoid effects on memory. Repeated exposure to the testing arena might be mildly stressful to the animals. Prior evidence indicated that a history of repeated (restraint) stress reduces CB1 receptor function on GABAergic terminals within several brain regions involved in learning and memory (Hu et al, 2011; Patel et al, 2009). As such, the divergent effects of WIN55,212-2 on short-term retention of rats in the WITHOUT-habituation and WITH-habituation groups may be due to a different ability of $\mathrm{CB} 1$ receptor activation to suppress GABAergic inhibitory control because of the stress history of the animals and not the immediate levels of arousal during the training session. In both scenarios, however, our findings show that the cannabinoid system exerts an environmentally sensitive modulation of short-term memory. As WIN55,212-2 levels are probably still elevated at the time of the 1-h retention test, it is most likely that WIN55,212-2 affected short-term retention performance via direct influences on the retrieval of memory processing (Atsak et al, 2012a).

Whereas clinical studies mainly focused on the potentially disruptive effects of cannabinoid drugs on short-term cognitive performance, findings of animal experiments provide extensive evidence that cannabinoid treatments also influence long-term memory. However, findings of these experiments have been conflicting as well. Some studies reported that cannabinoid agonists impair the consolidation of long-term memory of training on several tasks (Barros et al, 2004; Robinson et al, 2008), whereas others found enhancing effects (De Oliveira Alvares et al, 2008). Differences in dosage or drug administration regimen (eg, pretraining $v s$ posttraining administration) could have contributed to these opposite findings. Here we show that, similar to WIN55,212-2 effects on short-term memory, the level of emotional arousal at encoding, or alternatively the stress history of the animals, is another important factor modulating cannabinoid effect on long-term memory. WIN55,212-2 enhanced 24-h retention of rats that were not previously habituated to the experimental context, but failed to significantly alter performance of well-habituated rats. As WIN55,212-2 was administered immediately after the training trial, the effect of WIN55,212-2 on long-term retention is likely mediated by a selective influence on the consolidation of memory (McGaugh and Roozendaal, 2002). Moreover, because retention testing took place $24 \mathrm{~h}$ after the training and drug treatment, it is unlikely that the effects are mediated by any residual pharmacological effects on directly influencing behavior during retention testing (Cahill et al, 2001).

Clinical evidence supports the view that cannabis consumption can induce opposite effects on a variety of behaviors and subjective feelings in different individuals or even in the same subject (Green et al, 2003). Apart from genetic vulnerability (Xian et al, 2008), environmental factors such as stress-induced alterations could contribute to these differential effects (Viveros et al, 2012; Xian et al,
2008). Preclinical models have confirmed opposite effects of cannabinoid drugs on motor activity (Fonseca et al, 1998), positive incentive and/or motivational processes (Chaperon and Thiebot, 1999), anxiety (Haller et al, 2009) and fearrelated behaviors (Moreira and Wotjak, 2011). Although differences in dose, route of administration, and timing of exposure are typically listed as being responsible for the opposite behavioral effects, recent findings indicate that variations in the stressfulness of the experimental conditions employed in the different studies are implicated as well. The general assumption that enhancement of cannabinoid levels via an inhibition of the anandamide-degrading enzyme fatty acid amide hydrolase (FAAH) results in anxiolytic- and antidepressant-like effects in rodents (Gobbi et al, 2005; Kathuria et al, 2003) has recently been revisited. Haller et al (2009) found robust anxiolytic-like effects of the FAAH inhibitor URB597 when rats were tested without prior habituation to the experimental room. However, URB597 administration did not induce any anxiolytic-like effect in rats that were habituated to the testing environment. Similarly, both low and high doses of delta9tetrahydrocannabinol, the main psychoactive constituent of cannabis preparations, exerted anxiolytic-like effects in non-stressed animals (Fokos and Panagis, 2010). In stressed animals, however, only the higher dose of delta9-tetrahydrocannabinol induced an anxiolytic-like response whereas the lower dose induced an anxiogenic effect. Our present findings are thus very reminiscent of these other behaviors and indicate that the level of emotional arousal that is associated with the training procedure is also a key regulator of cannabinoid effects on both short- and longterm recognition memory.

Considering the extensive evidence indicating a close relationship between the cannabinoid system and HPA-axis activity (Armario, 2010; Barna et al, 2004), we explored the possibility that the divergent effects of systemic WIN55,2122 administration on object recognition memory might be related to differential effects of WIN55,212-2 on traininginduced glucocorticoid levels in rats in these two habituation conditions. Our findings indicate that WIN55,212-2 administration produced opposite effects on traininginduced plasma corticosterone levels in habituated $v s$ non-habituated rats. Generally, these findings are thus consistent with the evidence that the cannabinoid system is an important regulator of HPA-axis activity and consequently of the release of glucocorticoid hormones (Cota et al, 2007; Ganon-Elazar and Akirav, 2009). Our finding that WIN55,212-2 induced opposite effects on HPA-axis activity in rats in the two habituation conditions has a twofold interest. First, these findings might help to reconcile earlier observations that cannabinoid administration can both activate and inhibit the HPA-axis (Cota et al, 2007; Ganon-Elazar et al, 2009). Second, as extensive evidence indicates that glucocorticoid hormones influence both short- and long-term memory of emotionally arousing experiences (Roozendaal et al, 2009; Schwabe et al, 2012), these findings suggest the intriguing possibility that this arousal-dependent influence of WIN55,212-2 on the HPAaxis might contribute to the observed opposite effects of WIN55,212-2 on both short- and long-term memory. Highly comparable to our present findings with WIN55,212-2, it has been previously reported that corticosterone 
administration after object recognition training impaired short-term performance in emotionally aroused nonhabituated rats (Okuda et al, 2004; Roozendaal et al, 2006b). Moreover, corticosterone enhanced 24-h retention performance of rats that were not previously habituated to the experimental context. Corticosterone administration to well-habituated rats failed to affect either short- or longterm object recognition performance. Even though the dose of WIN55,212-2 that affected memory function in the non-habituated rats did not overlap entirely with its efficacious dose on corticosterone levels, our assumption was confirmed by the finding that adrenocortical suppression with the corticosterone-synthesis inhibitor metyrapone in non-habituated rats altered the effect of posttraining WIN55,212-2 administration on both short- and long-term recognition memory in such a way that performance became similar to that seen in habituated animals. Thus, these findings provide strong support for the view that a WIN55,212-2-induced potentiation of glucocorticoid secretion plays an important role in determining the pattern of cannabinoid effects on memory in these animals.

Previously, we showed that the cannabinoid and glucocorticoid effects on memory might converge within the basolateral amygdala (BLA). The possible interaction of these two systems was originally investigated for memory consolidation of inhibitory avoidance training (Campolongo et al, 2009) but similar mechanisms might exist in other brain regions for regulating other memory functions (Atsak et al, 2012a). Extensive evidence indicates that the BLA preferentially modulates memory of emotionally arousing training experiences (McGaugh, 2000) and that arousal-induced activation of noradrenergic signaling plays an important role herein (Quirarte et al, 1997; Roozendaal et al, 2002, 2004, 2006a). In prior studies, we showed that training-induced noradrenergic activity within the BLA is required for enabling the modulatory influence of glucocorticoids on memory (Quirarte et al, 1997). As habituation to the object recognition apparatus is known to attenuate training-induced increases in noradrenergic activity (Roozendaal et al, 2006b), we previously hypothesized that glucocorticoids might not modulate memory of object recognition training in habituated rats because of inadequate levels of arousal-induced norepinephrine. In support of this interpretation, we found that stimulating noradrenergic activity with the $\alpha_{2}$-adrenoceptor antagonist yohimbine was sufficient to enable glucocorticoid-induced memory enhancement in habituated rats (Roozendaal et al, 2006b). Recent findings indicate that the endocannabinoid system within the BLA might be importantly involved in regulating glucocorticoid effects on memory (Campolongo et al, 2009). Systemically administered corticosterone rapidly elevates endocannabinoid levels in the amygdala (Hill et al, 2010a), whereas a blockade of CB1 receptor activity in the BLA prevents corticosterone-induced memory enhancement (Campolongo et al, 2009). On the basis of these findings, we previously proposed a model in which glucocorticoids bind to a membrane-bound receptor in the BLA that activates a G-protein signaling cascade to stimulate the synthesis of endocannabinoids. The ensuing release of endocannabinoid ligands could diffuse to local GABAergic terminals and inhibit GABA release onto noradrenergic terminals in the BLA (Campolongo et al,
2009; Hill and McEwen, 2009). Importantly, as recent findings indicate that the $\beta$-adrenoceptor antagonist propranolol infused into the BLA also prevented the memory-enhancing effect of WIN55,212-2 (Hauer et al, 2010), it is thus possible that, comparable to the effects of glucocorticoids, cannabinoid effects on memory are also dependent on the availability of sufficient levels of arousalinduced noradrenergic activity within the BLA. On top of this direct interaction of WIN55,212-2 with arousal-induced noradrenergic mechanisms within the brain, the opposite effects of WIN55,212-2 on circulating corticosterone levels might add to the environmentally sensitive effects of cannabinoids on both short- and long-term recognition memory.

In summary, we provide evidence that cannabinoid effects on short- and long-term retention of recognition memory depend on the level of novelty-induced emotional arousal. These cannabinoid effects likely involve, at least in part, peripheral actions on modulating HPA-axis activity. These findings shed light on the contrasting effects of cannabinoid drugs on memory processing, thus providing new evidence that cannabinoid compounds can be either beneficial or detrimental to memory processes depending on the affective state of the individual at the time of drug consumption.

\section{ACKNOWLEDGEMENTS}

We thank Daniela Valeri and Veronica Carrara for technical help. This study was supported by grants from Sapienza University 'Progetti di Ricerca di Università', Vigoni Project (Ateneo Italo Tedesco) to PC and GS and from MIUR, FIRB Futuro in Ricerca to PC.

\section{DISCLOSURE}

The authors declare no conflict of interest.

\section{REFERENCES}

Armario A (2010). Activation of the hypothalamic-pituitaryadrenal axis by addictive drugs: different pathways, common outcome. Trends Pharmacol Sci 31: 318-325.

Atsak P, Hauer D, Campolongo P, Schelling G, McGaugh JL, Roozendaal B (2012a). Glucocorticoids interact with the hippocampal endocannabinoid system in impairing retrieval of contextual fear memory. Proc Natl Acad Sci USA 109: 3504-3509.

Atsak P, Roozendaal B, Campolongo P (2012b). Role of the endocannabinoid system in regulating glucocorticoid effects on memory for emotional experiences. Neuroscience 204: 104-116.

Baek J, Zheng Y, Darlington CL, Smith PF (2009). The CB1 receptor agonist, WIN 55,212-2, dose-dependently disrupts object recognition memory in adult rats. Neurosci Lett 464: 71-73.

Barna I, Zelena D, Arszovszki AC, Ledent C (2004). The role of endogenous cannabinoids in the hypothalamo-pituitary-adrenal axis regulation: in vivo and in vitro studies in $\mathrm{CB} 1$ receptor knockout mice. Life Sci 75: 2959-2970.

Barros DM, Carlis V, Maidana M, Silva ES, Baisch AL, Ramirez MR et al (2004). Interactions between anandamide-induced anterograde amnesia and post-training memory modulatory systems. Brain Res 1016: 66-71. 
Cahill L, McGaugh JL, Weinberger NM (2001). The neurobiology of learning and memory: some reminders to remember. Trends Neurosci 24: 578-581.

Campolongo P, Ratano P, Manduca A, Scattoni ML, Palmery M, Trezza V et al (2012). The endocannabinoid transport inhibitor AM404 differentially modulates recognition memory in rats depending on environmental aversiveness. Front Behav Neurosci 6: 11 .

Campolongo P, Roozendaal B (2011). Stress and glucocorticoid effects on memory. In: Conrad CD (ed) New Edition: Handbook of Stress: Neuropsychological Effects on the Brain. Wiley-Blackwell: Malden.

Campolongo P, Roozendaal B, Trezza V, Hauer D, Schelling G, McGaugh JL et al (2009). Endocannabinoids in the rat basolateral amygdala enhance memory consolidation and enable glucocorticoid modulation of memory. Proc Natl Acad Sci USA 106: 4888-4893.

Carlin AS, Bakker CB, Halpern L, Post RD (1972). Social facilitation of marijuana intoxication: impact of social set and pharmacological activity. J Abnorm Psychol 80: 132-140.

Chaperon F, Thiebot MH (1999). Behavioral effects of cannabinoid agents in animals. Crit Rev Neurobiol 13: 243-281.

Clarke JR, Rossato JI, Monteiro S, Bevilaqua LR, Izquierdo I, Cammarota M (2008). Posttraining activation of CB1 cannabinoid receptors in the CA1 region of the dorsal hippocampus impairs object recognition long-term memory. Neurobiol Learn Mem 90: 374-381.

Cota D, Steiner MA, Marsicano G, Cervino C, Herman JP, Grubler $\mathrm{Y}$ et al (2007). Requirement of cannabinoid receptor type 1 for the basal modulation of hypothalamic-pituitary-adrenal axis function. Endocrinology 148: 1574-1581.

De Oliveira AlvaresL, Genro BP, Diehl F, Quillfeldt JA (2008). Differential role of the hippocampal endocannabinoid system in the memory consolidation and retrieval mechanisms. Neurobiol Learn Mem 90: 1-9.

Deadwyler SA, Goonawardena AV, Hampson RE (2007). Shortterm memory is modulated by the spontaneous release of endocannabinoids: evidence from hippocampal population codes. Behav Pharmacol 18: 571-580.

Di S, Malcher-Lopes R, Halmos KC, Tasker JG (2003). Nongenomic glucocorticoid inhibition via endocannabinoid release in the hypothalamus: a fast feedback mechanism. J Neurosci 23: 4850-4857.

Emmert MH, Herman JP (1999). Differential forebrain c-fos mRNA induction by ether inhalation and novelty: evidence for distinctive stress pathways. Brain Res 845: 60-67.

Feenstra MG, Botterblom MH, Mastenbroek S (2000). Dopamine and noradrenaline efflux in the prefrontal cortex in the light and dark period: effects of novelty and handling and comparison to the nucleus accumbens. Neuroscience 100: 741-748.

Fokos S, Panagis G (2010). Effects of delta9-tetrahydrocannabinol on reward and anxiety in rats exposed to chronic unpredictable stress. J Psychopharmacol 24: 767-777.

Ganon-Elazar E, Akirav I (2009). Cannabinoid receptor activation in the basolateral amygdala blocks the effects of stress on the conditioning and extinction of inhibitory avoidance. J Neurosci 29: 11078-11088.

Gobbi G, Bambico FR, Mangieri R, Bortolato M, Campolongo P, Solinas $M$ et al (2005). Antidepressant-like activity and modulation of brain monoaminergic transmission by blockade of anandamide hydrolysis. Proc Natl Acad Sci USA 102: $18620-18625$

Green B, Kavanagh D, Young R (2003). Being stoned: a review of self-reported cannabis effects. Drug Alcohol Rev 22: 453-460.

Grota LJ, Bienen T, Felten DL (1997). Corticosterone responses of adult Lewis and Fischer rats. J Neuroimmunol 74: 95-101.

Haller J, Barna I, Barsvari B, Gyimesi Pelczer K, Yasar S, Panlilio LV et al (2009). Interactions between environmental aversiveness and the anxiolytic effects of enhanced cannabinoid signaling by FAAH inhibition in rats. Psychopharmacology (Berl) 204: 607-616.

Handa RJ, Nunley KM, Lorens SA, Louie JP, McGivern RF, Bollnow MR (1994). Androgen regulation of adrenocorticotropin and corticosterone secretion in the male rat following novelty and foot shock stressors. Physiol Behav 55: 117-124.

Hauer A, Fornari R, Atsak P, Campolongo P, Schelling G, Roozendaal R (2010). Memory consolidation via membrane glucocorticoid receptors that can be blocked by cannabinoid receptor antagonists. Eur Neuropsychopharmacol (2010; 20: S283-S284.

Hill MN, Karatsoreos IN, Hillard CJ, McEwen BS (2010a). Rapid elevations in limbic endocannabinoid content by glucocorticoid hormones in vivo. Psychoneuroendocrinology 35: 1333-1338.

Hill MN, McEwen BS (2009). Endocannabinoids: The silent partner of glucocorticoids in the synapse. Proc Natl Acad Sci USA 106: 4579-4580.

Hill MN, Patel S, Campolongo P, Tasker JG, Wotjak CT, Bains JS (2010b). Functional interactions between stress and the endocannabinoid system: from synaptic signaling to behavioral output. J Neurosci 30: 14980-14986.

Hu W, Zhang M, Czeh B, Zhang W, Flugge G (2011). Chronic restraint stress impairs endocannabinoid mediated suppression of GABAergic signaling in the hippocampus of adult male rats. Brain Res Bull 85: 374-379.

Kathuria S, Gaetani S, Fegley D, Valino F, Duranti A, Tontini A et al (2003). Modulation of anxiety through blockade of anandamide hydrolysis. Nat Med 9: 76-81.

Kosiorek P, Hryniewicz A, Bialuk I, Zawadzka A, Winnicka MM (2003). Cannabinoids alter recognition memory in rats. Pol J Pharmacol 55: 903-910.

Marsicano G, Lafenetre P (2009). Roles of the endocannabinoid system in learning and memory. Curr Top Behav Neurosci 1: 201-230.

McGaugh JL (2000). Memory-a century of consolidation. Science 287: $248-251$.

McGaugh JL, Roozendaal B (2002). Role of adrenal stress hormones in forming lasting memories in the brain. Curr Opin Neurobiol 12: 205-210.

McQuade R, Creton D, Stanford SC (1999). Effect of novel environmental stimuli on rat behaviour and central noradrenaline function measured by in vivo microdialysis. Psychopharmacology (Berl) 145: 393-400.

Moreira FA, Wotjak CT (2011). Cannabinoids and anxiety. Curr Top Behav Neurosci 2: 429-450.

Okuda S, Roozendaal B, McGaugh JL (2004). Glucocorticoid effects on object recognition memory require training-associated emotional arousal. Proc Natl Acad Sci USA 101: 853-858.

Pamplona FA, Bitencourt RM, Takahashi RN (2008). Short- and long-term effects of cannabinoids on the extinction of contextual fear memory in rats. Neurobiol Learn Mem 90: 290-293.

Patel S, Kingsley PJ, Mackie K, Marnett LJ, Winder DG (2009). Repeated homotypic stress elevates 2-arachidonoylglycerol levels and enhances short-term endocannabinoid signaling at inhibitory synapses in basolateral amygdala. Neuropsychopharmacology 34: 2699-2709.

Pattij T, Wiskerke J, Schoffelmeer AN (2008). Cannabinoid modulation of executive functions. Eur $J$ Pharmacol 585: 458-463.

Piomelli D (2003). The molecular logic of endocannabinoid signalling. Nat Rev Neurosci 4: 873-884.

Quirarte GL, Roozendaal B, McGaugh JL (1997). Glucocorticoid enhancement of memory storage involves noradrenergic activation in the basolateral amygdala. Proc Natl Acad Sci USA 94: 14048-14053.

Robinson L, McKillop-Smith S, Ross NL, Pertwee RG, Hampson RE, Platt B et al (2008). Hippocampal endocannabinoids inhibit 
spatial learning and limit spatial memory in rats. Psychopharmacology (Berl) 198: 551-563.

Rodriguez de, Fonseca F, Del Arco I, Martin-Calderon JL, Gorriti MA, Navarro M (1998). Role of the endogenous cannabinoid system in the regulation of motor activity. Neurobiol Dis 5(6 Pt B): 483-501.

Roozendaal B (2002). Stress and memory: opposing effects of glucocorticoids on memory consolidation and memory retrieval. Neurobiol Learn Mem 78: 578-595.

Roozendaal B, de Quervain DJ, Schelling G, McGaugh JL (2004). A systemically administered beta-adrenoceptor antagonist blocks corticosterone-induced impairment of contextual memory retrieval in rats. Neurobiol Learn Mem 81: 150-154.

Roozendaal B, Hui GK, Hui IR, Berlau DJ, McGaugh JL, Weinberger NM (2006a). Basolateral amygdala noradrenergic activity mediates corticosterone-induced enhancement of auditory fear conditioning. Neurobiol Learn Mem 86: 249-255.

Roozendaal B, McEwen BS, Chattarji S (2009). Stress, memory and the amygdala. Nat Rev Neurosci 10: 423-433.

Roozendaal B, Okuda S, Van der Zee EA, McGaugh JL (2006b). Glucocorticoid enhancement of memory requires arousalinduced noradrenergic activation in the basolateral amygdala. Proc Natl Acad Sci USA 103: 6741-6746.

Roozendaal B, Quirarte GL, McGaugh JL (2002). Glucocorticoids interact with the basolateral amygdala beta-adrenoceptorcAMP/cAMP/PKA system in influencing memory consolidation. Eur J Neurosci 15: 553-560.

Saghafi N, Lam DK, Schmidt BL (2011). Cannabinoids attenuate cancer pain and proliferation in a mouse model. Neurosci Lett 488: 247-251.

Schimmer BP, Parker KL (2001). Goodman \& Gilman's The Pharmacological Basis of Therapeutics10th edition In: Hardman Joel GLLEAlfred GoodmanGilman (ed) Goodman \& Gilman's The Pharmacological Basis of Therapeutics 10th edition. McGraw-Hill, p 1675.
Schneider M, Schomig E, Leweke FM (2008). Acute and chronic cannabinoid treatment differentially affects recognition memory and social behavior in pubertal and adult rats. Addict Biol 13: 345-357.

Schwabe L, Joels M, Roozendaal B, Wolf OT, Oitzl MS (2012). Stress effects on memory: an update and integration. Neurosci Biobehav Rev 36: 1740-1749.

Strashimirov D, Bohus B (1966). Effect of 2-methyl-1,2-bis-3pyridl-1-propanone (SU-4885) on adrenocortical secretion in normal and hypophysectomized rats. Steroids 7: 171-180.

Suenaga T, Ichitani Y (2008). Effects of hippocampal administration of a cannabinoid receptor agonist WIN 55,212-2 on spontaneous object and place recognition in rats. Behav Brain Res 190: 248-252.

Szuster RR, Pontius EB, Campos PE (1988). Marijuana sensitivity and panic anxiety. J Clin Psychiatry 49: 427-429.

van den Buuse M, Van Acker SA, Fluttert M, De Kloet ER (2001). Blood pressure, heart rate, and behavioral responses to psychological 'novelty' stress in freely moving rats. Psychophysiology 38: 490-499.

Viveros MP, Llorente R, Suarez J, Llorente-Berzal A, Lopez-Gallardo M, Rodriguez de FonsecaF (2012). The endocannabinoid system in critical neurodevelopmental periods: sex differences and neuropsychiatric implications. J Psychopharmacol 26: $164-176$

Wilson RI, Nicoll RA (2002). Endocannabinoid signaling in the brain. Science 296: 678-682.

Xian H, Scherrer JF, Grant JD, Eisen SA, True WR, Jacob T et al (2008). Genetic and environmental contributions to nicotine, alcohol and cannabis dependence in male twins. Addiction 103: 1391-1398.

Zanettini C, Panlilio LV, Alicki M, Goldberg SR, Haller J, Yasar S (2011). Effects of endocannabinoid system modulation on cognitive and emotional behavior. Front Behav Neurosci 5: 57. 